\title{
Coupling Bright and Dark Plasmonic Lattice Resonances
}

\author{
S. R. K. Rodriguez, ${ }^{1, *}$ A. Abass, ${ }^{2}$ B. Maes, ${ }^{3}$ O. T. A. Janssen, ${ }^{4}$ G. Vecchi, ${ }^{1}$ and J. Gómez Rivas ${ }^{1,5}$ \\ ${ }^{1}$ Center for Nanophotonics, FOM Institute AMOLF, clo Philips Research Laboratories, High Tech Campus 4, \\ 5656 AE Eindhoven, The Netherlands \\ ${ }^{2}$ Department of Electronic and Information Systems (ELIS), Ghent University, Sint-Pietersnieuwstraat 41, B-9000 Ghent, Belgium \\ ${ }^{3}$ Micro- and Nanophotonic Materials Group, Institut de Physique, University of Mons, Place du Parc 20, B-7000 Mons, Belgium \\ ${ }^{4}$ Optics Research Group, Delft University of Technology, 2628 CJ Delft, The Netherlands \\ ${ }^{5}$ Department of Applied Physics, Eindhoven University of Technology, P.O. Box 513, 5600 MB Eindhoven, The Netherlands
}

(Received 25 March 2011; published 15 December 2011)

\begin{abstract}
We demonstrate the coupling of bright and dark surface lattice resonances (SLRs), which are collective Fano resonances in 2D plasmonic crystals. As a result of this coupling, a frequency stop gap in the dispersion relation of SLRs is observed. The different field symmetries of the low- and high-frequency SLR bands lead to pronounced differences in their coupling to free-space radiation. Standing waves of very narrow spectral width compared to localized surface-plasmon resonances are formed at the highfrequency band edge, while subradiant damping onsets at the low-frequency band edge, leading the resonance into darkness. We introduce a coupled-oscillator analog to the plasmonic crystal, which serves to elucidate the physics of the coupled plasmonic resonances and which is used to estimate very high quality factors for SLRs.
\end{abstract}

DOI: 10.1103/PhysRevX.1.021019

Metallic nanoparticles supporting surface-plasmon resonances allow light to be localized in nanoscale volumes, thereby opening exciting possibilities such as nanoscale control of emitters [1], large electromagnetic enhancements [2], and nonlinear nano-optics [3]. Much attention has been given to localized surface-plasmon resonances (LSPRs), which arise in individual particles when their conduction electrons are coherently driven by an electromagnetic field. Although localized surface plasmons in neighbor particles may mutually couple, their resonances are, in general, severely broadened due to strong radiative damping. Hence, LSPRs exhibit low quality factors $Q$. A recent development in nanoplasmonics deals with collective resonances in periodic arrays of metallic nanostructures, or plasmonic crystals. Such arrays support surface lattice resonances (SLRs), which are collective resonances mediated by diffractive coupling of localized plasmons. This coupling occurs near the critical frequency at which a diffraction order is radiating in the plane of the array, i.e., at the Rayleigh anomaly. SLRs were introduced by Carron [4], and the interest in this phenomenon was revived by Schatz and co-workers with a series of works on $1 \mathrm{D}$ and 2D arrays $[5,6]$. However, the experimental observation of SLRs remained elusive for many years [7]. Recent advances in nanofabrication and in the understanding of SLRs have allowed for their observation in periodic arrays of nanostructures with different geometries [8-13]. In contrast

\footnotetext{
*s.rodriguez@amolf.nl
}

Published by the American Physical Society under the terms of the Creative Commons Attribution 3.0 License. Further distribution of this work must maintain attribution to the author(s) and the published article's title, journal citation, and DOI.
Subject Areas: Nanophysics, Plasmonics

with LSPRs, SLRs possess much higher $Q$ 's, and the associated polaritons can propagate over tens of unit cells in the plasmonic crystal [12]. The relevance of SLRs for enhanced, directional, and polarized light emission [11,14] and sensing [15] has been recently demonstrated. Although the coupling of surface modes in periodic metallic structures has attracted much interest [16-19], especially for its connection with frequency stop gaps [20], coupled SLRs have not yet been discussed.

In this paper, we demonstrate the mutual coupling of SLRs and the formation of a frequency stop gap in the dispersion relation of these modes. This coupling leads to a strong modification of the SLRs characteristics, including the onset of subradiant damping in the low-frequency band, zero group-velocity modes in the high-frequency band, and $Q$ factors for both bands, which are amongst the highest reported for any $2 \mathrm{D}$ plasmonic crystal. Our results set the basis for controlling the dispersion of SLRs, and they open new possibilities in sensing, enhanced spontaneous light emission, and lasing at the band edges of SLR gaps.

We have investigated $3 \times 3 \mathrm{~mm}^{2}$ arrays of gold nanorods fabricated on a silica substrate using substrate conformal imprint lithography [21]. The insets of Figs. 1(a) and 1(b) show top-view SEM images of arrays with lattice constants $a_{x}=600 \mathrm{~nm}$ and $a_{y}=300 \mathrm{~nm}$ in both cases, and rods of dimensions (a) $420 \times 85 \times 40 \mathrm{~nm}^{3}$ and (b) $450 \times 120 \times 38 \mathrm{~nm}^{3}$. The arrays were embedded in a uniform surrounding medium by placing a silica superstrate preceded with $n=1.45$ index matching fluid to ensure good optical contact. We measured the variableangle zeroth-order transmittance of the collimated beam from a halogen lamp while rotating the samples around the $y$ axis. The polarization of the incident light was set along 


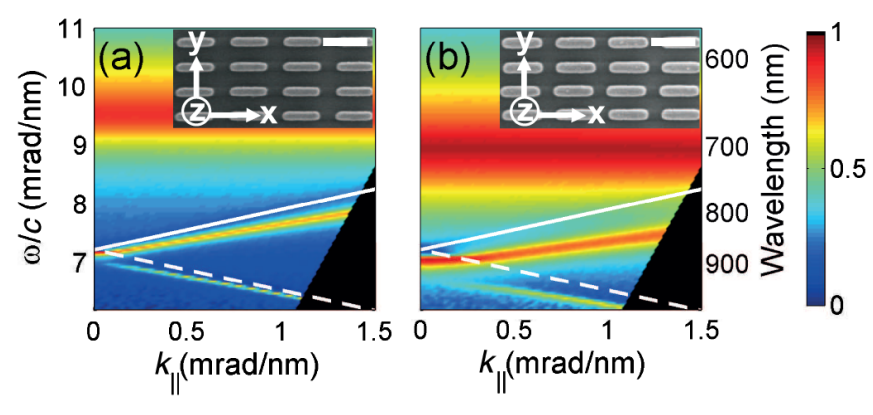

FIG. 1. Extinction spectra as a function of $k_{\|}$for an array of gold nanorods of dimensions (a) $420 \times 85 \times 40 \mathrm{~nm}^{3}$ and (b) $450 \times 120 \times 38 \mathrm{~nm}^{3}$. The insets of (a) and (b) display scanning electron micrographs of the corresponding arrays; the scale bars denote $500 \mathrm{~nm}$. The incident light is polarized along the y direction, probing the short axis of the nanorods. The lattice constants are $a_{x}=600 \mathrm{~nm}$ and $a_{y}=300 \mathrm{~nm}$ for both arrays. The solid and dashed white lines representing the $(+1,0)$ and $(-1,0)$ Rayleigh anomalies, respectively, are equal in both cases. The dispersionless resonances on the high-frequency side of the spectra are the dipolar localized surface-plasmon resonances for the short axis of the nanorods, whereas the narrower and dispersive resonances below the Rayleigh anomalies are the surface lattice resonances.

the $y$ axis, probing the short axis of the nanorods. For this polarization, the dipolar LSPR lies at higher energies than the $( \pm 1,0)$ diffraction orders, thereby allowing the coupling of localized surface plasmons to these orders [8].

Figure 1 displays the extinction of the arrays defined as $1-T_{0}$, with $T_{0}$ the zeroth-order transmittance. The measurements are shown as a function of the reduced frequency, i.e., the angular frequency normalized by the speed of light in vacuum, and the projection of the incident wave vector onto the surface of the array $k_{\|}=\frac{\omega}{c} \sin (\theta) \hat{x}$, with $\theta$ the angle of incidence. The broad, dispersionless resonance on the high-frequency side of both spectra corresponds to the excitation of dipolar LSPRs in individual nanorods. The $(+1,0)$ and $(-1,0)$ Rayleigh anomalies, which are the conditions for which the corresponding diffracted orders are grazing to the surface, are indicated by the solid and dashed white lines in Fig. 1, respectively. The coupling of localized surface plasmons to the Rayleigh anomalies leads to sharp peaks in extinction on the lowfrequency side of the Rayleigh anomalies. These peaks correspond to the excitation of SLRs. Notice that for the wider nanorods [Fig. 1(b)], the LSPR is shifted down in frequency and spectrally broadened - the expected behavior due to increased depolarization and radiative damping [22]. This lower detuning between the LSPR and the $( \pm 1,0)$ Rayleigh anomalies increases the radiative coupling of localized surface plasmons to diffracted orders. As we will explain ahead, in terms of a coupled-oscillator analog to the plasmonic crystal, an increased coupling strength is responsible for a higher detuning of the SLRs from their associated Rayleigh anomalies, and for a broader SLR linewidth. Notice also that a frequency stop gap is formed for both arrays near $\omega / c=7 \mathrm{mrad} / \mathrm{nm}$ and $k_{\|}=0$, where the two SLRs mutually couple, and that the gap becomes wider in Fig. 1(b). It is clear from Fig. 1 that the properties of SLRs, i.e., their dispersion, center frequency, linewidth, and mutual coupling, are strongly influenced by the interplay between the LSPR and the Rayleigh anomalies. A detailed explanation of this interplay is presented in Ref. [23]. In this work, we discuss the variableangle properties of coupled bright and dark SLRs, focusing on the measurements in Fig. 1(b).

Figure 1(b) shows the formation of a stop gap centered at $\omega / c=6.85 \mathrm{mrad} / \mathrm{nm}$ and near $k_{\|}=0$. We note that this is not a complete photonic band gap, since it exists for $y$-polarized light only. The gap arises from the coupling of two counterpropagating surface polaritons, which, due to the structural anisotropy of both the nanorods and the lattice, have a strong polarization dependence on their coupling to free-space radiation. At the high-frequency band edge, we observe that the dispersion of the $(+1,0)$ SLR flattens. This flattening of the band can be translated as a reduction of the mode's group velocity and the formation of standing waves, which are also associated with an increased density of optical states. At the low-frequency band edge, the $(-1,0)$ SLR becomes weaker and narrower. This behavior is characteristic of a mode tending towards subradiance, where radiative damping is suppressed in a collective state with an antisymmetric wave function [24]. As shown in nanoslit arrays [18], there is an intimate connection between subradiant damping and the opening of a gap in the dispersion relation of surface-plasmon polaritons (SPPs).

Figure 2(a) shows a close view of the stop gap in Fig. 1(b), and Fig. 2(b) shows results from finite-element simulations (COMSOL). For the simulations, we used a constant refractive index of 1.45 for silica, the permittivity of gold as given in Ref. [25], and Bloch-Floquet boundary conditions. The transmittance was calculated as the ratio between the transmitted power through the array and the incident power. In Figs. 2(c) and 2(d) we compare simulations with measurements at $k_{\|}=0$ and $k_{\|}=$ $0.4 \mathrm{mrad} / \mathrm{nm}$, respectively. By reproducing the measured dispersion of SLRs and the gap's central frequency and width, a good qualitative agreement between measurements and simulations is demonstrated. Discrepancies in the amplitude and spectral width of the resonances can be mainly attributed to differences between the simulated and fabricated geometries, especially near the corners. We have also calculated the variable-angle extinction spectra of the array in Fig. 1(b) with the coupled dipole model [5-7,15], which treats each particle as a radiating dipole and calculates the dipolar interactions of all particles in the array. The results obtained were in significant disagreement with the measurements and the simulations, and we therefore do not show the spectra here. This discrepancy arises from the 

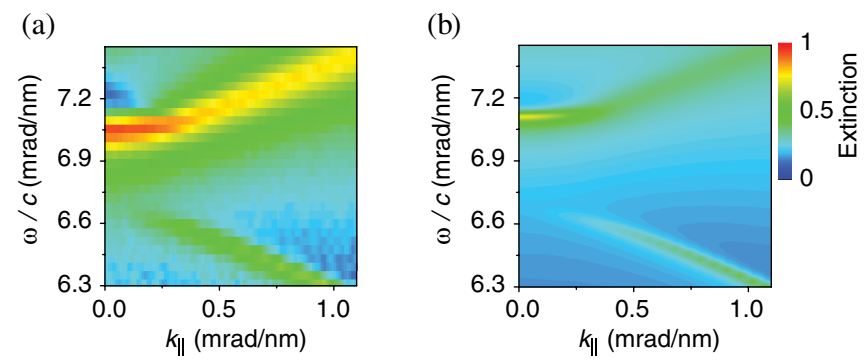

(c)

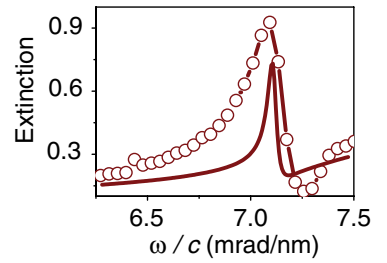

(d)

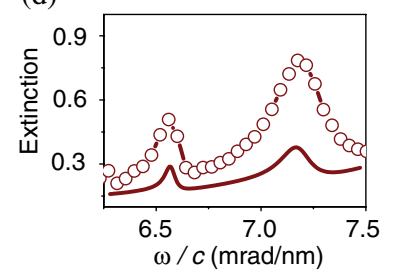

FIG. 2. Measurements (a) and finite-element simulations (b) of the extinction spectra of the array shown in Fig. 1. Panels (c) and (d) are cuts at $k_{\|}=0$ and $k_{\|}=0.4 \mathrm{mrad} / \mathrm{nm}$, respectively, of both (a) and (b). The open circles in (c) and (d) are measurements and the solid curves are simulations.

large size of the particles herein considered, which gives rise to multipolar contributions in the response, as we will show via the simulations results ahead.

It can be observed in Figs. 1 and 2 that the SLR peaks, together with the Rayleigh-anomaly dips, give rise to asymmetric resonance line shapes, which can be understood in the framework set forth by Fano [26]. Fano described the quantum interference between a discrete state and a continuum of states as the origin of asymmetric resonance line shapes. It was later shown in metallic subwavelength hole arrays that the coupling of surface plasmons to Rayleigh anomalies leads to similar line shapes, with the resonantly scattered light acting as the discrete state and the background transmission as the continuum [27]. A similar situation is observed in our configuration. The broad dipolar LSPR determines the extent of background transmission, i.e., the continuum, according to its frequency difference with the Rayleigh anomaly. The Rayleigh anomaly corresponds to the discrete state. The diffractive coupling of localized surface plasmons therefore resembles the interaction between a continuum of states and a discrete state, leading to asymmetric resonance line shapes. As seen in Figs. 1 and 2, the degree of asymmetry of these line shapes changes depending on the frequency and $k$-vector of excitation. This dependency is rooted in the relative contributions of resonant and nonresonant scattering [28], which also manifest as a modification of the SLR damping; the latter point will be addressed further in the text.

The different electrodynamic response leading to the bright and dark character-efficient and inefficient coupling to light —of the $(+1,0)$ and $(-1,0)$ SLRs, respectively, transpires from the near-field enhancement and (a)

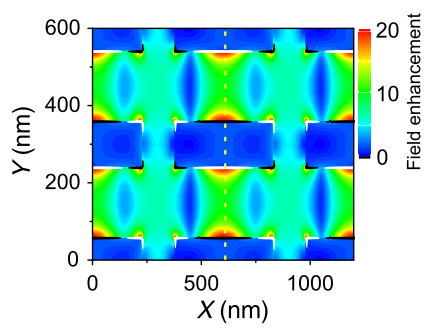

(b)

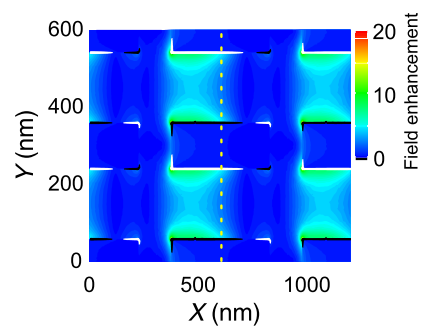

FIG. 3. Near-field enhancement in color scale and surfacecharge distribution (at an arbitrary phase) in black and white at the midheight of the nanorods for the (a) $(+1,0)$ and (b) $(-1,0)$ surface lattice resonance at $k_{\|}=0.15 \mathrm{mrad} / \mathrm{nm}$. The dotted lines indicate the plane of symmetry for coupling to radiation.

surface-charge distribution of the nanorods at the respective frequencies. In Fig. 3 we show simulation results for $k_{\|}=$ $0.15 \mathrm{mrad} / \mathrm{nm}$ at two frequencies: $\omega / c=7.1 \mathrm{mrad} / \mathrm{nm}$ and $\omega / c=6.7 \mathrm{mrad} / \mathrm{nm}$, which correspond to the $(+1,0)$ and $(-1,0)$ SLR, respectively. The small angle of incidence was chosen such that the extinction is not negligible for the $(-1,0)$ SLR. Both plots are at a plane parallel to the array located at the midheight of the nanorods. Charges of opposite sign at the surface of the nanorods are plotted in white and black, while the total near-field enhancement, i.e., $|E| /\left|E_{0}\right|$ with $E$ the total field and $E_{0}$ the incident field, is displayed by the color scale. Figure 3 illustrates how the different resonant response has its origin in the symmetry of the modes. In order to couple to the incident plane wave at normal incidence, the mode has to be symmetric with respect to the plane defined by the incident $k$ - and polarization vectors intersecting the nanorods along their center, i.e., the symmetry plane indicated by the dotted lines in Fig. 3. The $(+1,0)$ mode, which is shown in Fig. 3(a) for a small angle of incidence, has symmetric field and charge distributions with respect to the symmetry plane. A strong dipole moment is seen for each nanorod, and a strong dipolar interrod coupling takes places along the y direction also. This results in a large extinction and an efficient coupling of the $(+1,0)$ mode to normal incidence light, as observed in Fig. 1. In contrast, the $(-1,0)$ mode has an antisymmetric field and charge distribution for $k_{\|}=0$; the net dipole moment is therefore zero and the extinction vanishes. This symmetry is broken for angles of incidence larger than $\theta=0^{\circ}$, thus allowing the excitation of this resonant mode as shown in Fig. 3(b). The broken symmetry manifests as a quadrupolar surfacecharge distribution displaced from the symmetry axis. This results in a nonzero intrarod and interrod dipole moment, which can be recognized from the charges of opposite sign inside the nanorods and for adjacent nanorods along the symmetry axis, respectively.

The coupled nature of SLRs can be elucidated by making an analogy with a set of three mutually coupled harmonic oscillators. Coupled oscillators have proven useful 
in understanding electromagnetic phenomena [29,30]. In this analogy, the conduction electrons in the nanorods driven by the electromagnetic field are modeled as oscillator 1 , driven by a harmonic force $F=F_{0} e^{-i \omega_{s} t}$, whereas the $(+1,0)$ and $(-1,0)$ Rayleigh anomalies are modeled by oscillators 2 and 3, respectively. The equations of motion for the system are

$$
\begin{aligned}
& \ddot{x}_{1}+\gamma_{1} \dot{x}_{1}+\omega_{1}^{2} x_{1}-\Omega_{12}^{2} x_{2}-\Omega_{13}^{2} x_{3}=F, \\
& \ddot{x}_{2}+\gamma_{2} \dot{x}_{2}+\omega_{2}^{2} x_{2}-\Omega_{12}^{2} x_{1}-\Omega_{23}^{2} x_{3}=0, \\
& \ddot{x}_{3}+\gamma_{3} \dot{x}_{3}+\omega_{3}^{2} x_{3}-\Omega_{13}^{2} x_{1}-\Omega_{23}^{2} x_{2}=0,
\end{aligned}
$$

where $x_{j}, \gamma_{j}$, and $\omega_{j}(j=1,2,3)$ are the displacement from equilibrium position, damping, and eigenfrequency associated with the $j$ th oscillator, respectively, and $\Omega_{j k}$ ( $k=1,2,3$ and $j \neq k$ ) is the coupling frequency between the $j$ th and $k$ th oscillator. Since we are interested in the extinct optical power in driving the electrons in the nanorod, we calculate the absorbed mechanical power by oscillator 1 from the driving force, which is given by $P(t)=F \dot{x}_{1}$. Integrating $P(t)$ over one period of oscillation and scanning the driving frequency $\omega_{s}$ yield an absorbed power spectrum $P\left(\omega_{s}\right)$, which is representative of the extinction spectrum. Notice that Eq. (1) describes a coupled system of equations, whereby oscillators 2 and 3 are coupled to oscillator 1 , and therefore to $F$, via the coupling frequencies $\Omega_{12}$ and $\Omega_{13}$. Calculating $P\left(\omega_{s}\right)$ amounts to the total dissipated power by the first oscillator (the nanorod resonating at the LSPR) when this is driven by a harmonic force (the driving optical field) and coupled to two oscillators (the in-plane diffracted fields, i.e., the Rayleigh anomalies). From this simple mechanical analog, it transpires that the power dissipated by the nanorod is molded by the interference between the driving field and the diffracted fields.

In Fig. 4 we compare the real part of $P\left(\omega_{s}\right)$ with the measured extinction spectra at three values of $k_{\|}$. In all three cases $\omega_{1}=9.25 \mathrm{mrad} / \mathrm{nm}$ and $\gamma_{1}=2.3 \mathrm{mrad} / \mathrm{nm}$, which reproduce the frequency and damping of the LSPR, and $F_{0}=0.57$ units of force per mass, is a fitting parameter determining the amplitude of the spectra. The eigenfrequencies $\omega_{2}$ and $\omega_{3}$ are determined for each value of $k_{\|}$ by the corresponding $( \pm 1,0)$ Rayleigh anomalies of the array. As it will be shown ahead, a minimum in $P\left(\omega_{s}\right)$ arises at $\omega_{2}$ and $\omega_{3}$, implying that the Rayleigh-anomaly condition corresponds to a minimum in the dipolar moment of the particles. The remaining parameters, i.e., coupling and damping frequencies associated with the lattice modes, are the parameters used to fit the measured SLR line shapes and are given in Table I for three values of $k_{\|}$.

Figure 4(a) displays the spectra at $k_{\|}=0$, where the Rayleigh anomalies are degenerate at $\omega_{2}=$ $7.26 \mathrm{mrad} / \mathrm{nm}$ and the $(-1,0)$ SLR is a dark state. In this case, the model reduces to that of two coupled oscillators. With increasing $k_{\|}$the $(-1,0)$ SLR comes out of the

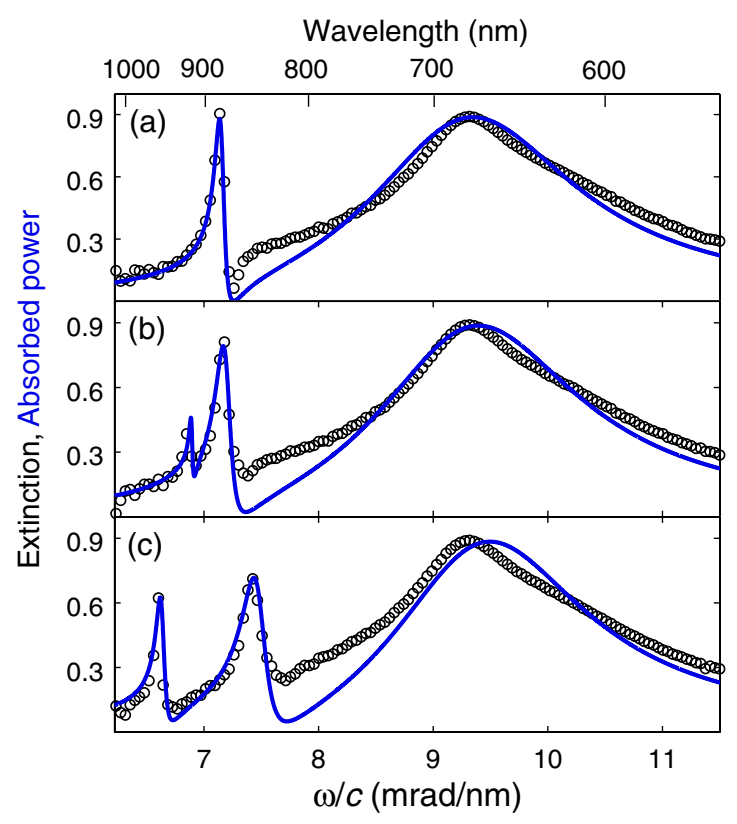

FIG. 4. The black open circles are cuts of the measured extinction spectra shown in Fig. 1 at three values of $k_{\|}$: (a) $k_{\|}=0 \mathrm{mrad} / \mathrm{nm}$, (b) $k_{\|}=0.17 \mathrm{mrad} / \mathrm{nm}$, and (c) $k_{\|}=$ $0.68 \mathrm{mrad} / \mathrm{nm}$. The blue solid curves represent the absorbed power in oscillator 1 of the coupled-oscillator model described in the text, with coupling and damping frequencies as given in Table I.

darkness, so the three oscillators are mutually coupled. In Fig. 4(b) we consider the case $k_{\|}=0.17 \mathrm{mrad} / \mathrm{nm}$, where $\omega_{2}=7.33 \mathrm{mrad} / \mathrm{nm}$ and $\omega_{3}=6.92 \mathrm{mrad} / \mathrm{nm}$. From the values given in Table I, we see that, with respect to the normal-incidence case, at $k_{\|}=0.17 \mathrm{mrad} / \mathrm{nm}$, the nanorods are more strongly coupled to the $(+1,0)$ Rayleigh anomaly ( $\Omega_{12}$ increases), and only weakly coupled to the $(-1,0)$ Rayleigh anomaly (low $\Omega_{13}$ ), the Rayleigh anomalies are mutually coupled (high $\Omega_{23}$ ), and the damping of both resonances has very significantly increased (both $\gamma_{2}$ and $\gamma_{3}$ increase). Further increasing to $k_{\|}=$ $0.68 \mathrm{mrad} / \mathrm{nm}$ gives $\omega_{2}=7.68 \mathrm{mrad} / \mathrm{nm}$ and $\omega_{3}=$ $6.70 \mathrm{mrad} / \mathrm{nm}$, which is the case in Fig. 4(c). At this value of $k_{\|}$, there is an increased coupling of the nanorods to both Rayleigh anomalies (both $\Omega_{12}$ and $\Omega_{13}$ increase), the coupling between the Rayleigh anomalies decreases $\left(\Omega_{23}\right.$ decreases), and the damping of both resonances increases (both $\gamma_{2}$ and $\gamma_{3}$ increase).

From the above-mentioned behaviors and the quantities given in Table I, we are able to draw several conclusions. First, the model shows how the coupling terms $\Omega_{j k}$ determine the frequency difference between the SLR peaks and the Rayleigh anomalies as evidenced in Fig. 1. Namely, for the $(+1,0)$ Rayleigh anomaly we observe that as $k_{\|}$increases, the SLR peak deviates more in frequency. This behavior is defined in the increase of $\Omega_{12}$, which detunes the resonance peak from the Rayleigh anomaly. In contrast, for the $(-1,0)$ diffraction order we observe a decreasing 
TABLE I. The $\Omega_{j k}$ terms $(j, k=1,2,3$, and $j \neq k)$ are the coupling frequencies between the $j$ th and $k$ th oscillators, and the $\gamma_{j}$ terms are the damping frequencies associated with the $j$ th oscillator, for the system described by Eq. (1). All quantities are given in units of $\mathrm{mrad} / \mathrm{nm}$. In the entries for which a minimum estimate is given, the value in parentheses represents the value yielding the spectra in Fig. 4.

\begin{tabular}{lccccc}
\hline \hline & $\Omega_{12}$ & $\Omega_{13}$ & $\Omega_{23}$ & $\gamma_{2}$ & $\gamma_{3}$ \\
\hline$k_{\|}=0$ & $2.8 \pm 0.1$ & $\ldots$ & $\ldots$ & $<0.01(0.001)$ & $\ldots$ \\
$k_{\|}=0.17$ & $3.1 \pm 0.1$ & $<0.6(0.1)$ & $1.1 \pm 0.1$ & $0.02 \pm 0.01$ & $0.020 \pm 0.005$ \\
$k_{\|}=0.68$ & $3.3 \pm 0.1$ & $2.4 \pm 0.1$ & $<0.7(0.3)$ & $0.06 \pm 0.02$ & $0.03 \pm 0.01$ \\
\hline \hline
\end{tabular}

frequency deviation of the SLR from its corresponding Rayleigh anomaly as $k_{\|}$increases. In this case, the dominant interaction near normal incidence is the mutual coupling of SLRs described by the term $\Omega_{23}$, which detunes the $(-1,0)$ SLR from its Rayleigh anomaly at low $k_{\|}$. Although $\Omega_{13}>\Omega_{23}$ at large values of $k_{\|}$, at low values of $k_{\|}$the $\Omega_{23}$ interaction dominates due to the smaller frequency difference between the eigenfrequencies $\omega_{3}$ and $\omega_{2}$. Second, the model shows that the damping of both resonances increases with $k_{\|}$, which leads to less asymmetric Fano line shapes and broader linewidths for both resonances. The decreasingly asymmetric line shapes as $k_{\|}$increases are especially clear in Fig. 4, whereas the SLR broadening and variable extinction of the Rayleigh anomalies are visible in Fig. 1.

Although the focus of this work has been on the variableangle SLR properties of one array, it can be deduced by comparing the measurements in Figs. 1(a) and 1(b) that the size of the nanorods plays a key role in exciting and molding the SLRs. As discussed in Ref. [23], this is done by changing the frequency and linewidth of the LSPR, i.e., $\omega_{1}$ and $\gamma_{1}$, in terms of the coupled-oscillator model, and the coupling strengths of the surface modes involved, i.e., the $\Omega_{j k}$ terms. We may summarize the details of these interactions as follows. For narrower nanorods where the LSPR lies at a higher frequency and the LSPR-Rayleigh-anomaly detuning is high, the SLRs attain a narrower linewidth, a lower extinction, and higher group velocity $v_{g}=\partial \omega / \partial k_{\|}$, following more closely the dispersion of the Rayleigh anomalies. This low coupling strength of the LSPR to the Rayleigh anomalies is expressed in the coupled-oscillator model as low values of $\Omega_{12}$ and $\Omega_{13}$. It follows from Eq. (1) that as $\Omega_{12}$ and $\Omega_{13}$ tend to zero and oscillators 2 and 3 become effectively decoupled, the extinction and linewidth associated with the SLRs vanish from the absorbed power spectrum by oscillator 1, leaving only the LSPR feature. This trend can be also observed in the measurements of Fig. 1, and it is further discussed in Ref. [23]. In contrast, for wider nanorods where the LSPR lies at a lower frequency and the LSPR-Rayleigh-anomaly detuning is low, the SLRs attain a broader linewidth, a higher extinction, and lower $v_{g}$. This higher LSPR-Rayleigh-anomaly coupling strength corresponds to increased values of $\Omega_{12}$ and $\Omega_{13}$. The variable properties of SLRs as a function of the
LSPR-Rayleigh anomaly coupling strengths are an expression of the polaritonic nature of SLRs [12]. Namely, SLRs acquire a photonlike character for large LSPR-Rayleighanomaly detunings, whereas a plasmonlike character is observed for low LSPR-Rayleigh-anomaly detunings. The diminishing SLR group velocity at the expense of a broader linewidth is, in simple terms, a manifestation of the well-known trade-off between propagation length and transverse confinement for surface polaritons.

It is interesting to calculate the $Q$ factors of the uncoupled oscillators, which follow from the definition $Q_{j}=$ $\omega_{j} / \gamma_{j}$. For oscillator 1 resonating at the LSPR frequency, we obtain $Q_{1}=4$. For oscillator 2 yielding the $(+1,0)$ SLR, we have $Q_{2}>700$ at normal incidence. This value, which is, to the best of our knowledge, higher than any reported $Q$ for an experimental study on 2D plasmonic crystals, is a minimum limit. This limit arises because for any value $\gamma_{2}<0.01$ a fit to the measurement within a $10 \%$ uncertainty in the magnitude of the extinction can be obtained. For oscillator 3 yielding the $(-1,0)$ SLR, we have $Q_{3}=300$ at $k_{\|}=0.17 \mathrm{mrad} / \mathrm{nm}$. It is important to realize that the resonances in the coupled system exhibit an effective damping, which is not equal to the damping of the uncoupled oscillators. Nevertheless, the above values clearly reflect the large differences in $Q$ factors between LSPRs and SLRs. Furthermore, the coupled-oscillator model points to the origin of the narrow SLR linewidths, which is the coupling of two harmonic oscillators with very different damping. By comparing $Q_{1}$ and $Q_{3}$ with previously reported experimental data, we get an insight into how well the $Q$ factors of the uncoupled oscillators represent the $Q$ factors of the resonances in the coupled system. First, $Q_{1}=4$ is a typical result for LSPRs with high radiative damping [22]. Second, in Ref. [18] Ropers and co-workers measured for subradiantly damped SPPs in a nanoslit array near stop gap [similar to what we observe for the $(-1,0)$ SLR near the gap] lifetimes near $\tau=200 \mathrm{fs}$. Nanoslits can be represented quite accurately as resonators [31], so the lifetime of the excited state $\tau$ is related to $Q$ by $Q=2 \pi \nu \tau$, with $\nu$ the frequency. Inserting $\tau=200$ fs and $\nu=400 \mathrm{THz}$ (the frequency of the subradiant mode in Ref. [18]) yields $Q=500$, which is comparable with the value that we find, i.e., $Q_{3}=300$. Despite the differences in the structures considered and possibly the extent of 
subradiant damping, it is remarkable that the oscillator model yields, at the very least, an order of magnitude estimate for the $Q$ of a nearly subradiant SPP mode in the vicinity of a stop gap. It should be mentioned that for arrays of dimensions on the order of $10 \mu \mathrm{m}$ or less, the $Q$ factors of SLRs are expected to appreciably decrease. This length scale corresponds to the associated surfacepolariton propagation lengths, which were recently determined in Ref. [12]. For arrays much larger than this characteristic length, such as the ones herein considered, the $Q$ factor saturates. The inhibition of radiative damping that leads to a narrowing of the SLR linewidth is therefore a collective effect, i.e., it depends on the number of particles present in the array.

In conclusion, we have demonstrated the coupling of SLRs and the associated opening of a frequency stop gap in the dispersion relation of these modes. The symmetric and antisymmetric field and charge distributions responsible for the bright and dark nature of the $(+1,0)$ and $(-1,0)$ modes, respectively, were illustrated. We have also estimated the very high quality factors of SLRs $(Q>700)$, which are, to the best of our knowledge, the highest reported values for any experimental study on 2D plasmonic crystals. Coupled bright and dark collective modes, as well as stop gaps, offer the possibility to carefully design abrupt changes in the local density of optical states (LDOS) over narrow spectral regions and in an extended volume. Local density of optical states manipulation in plasmonic structures is relevant for enhancing the efficiency of lightemitting devices, sensors, and nonlinear processes, all of which can be tailored in a frequency-, angle-, and polarization-dependent manner with coupled SLRs. Moreover, the standing waves formed at the highfrequency band edge hold exciting properties for the manipulation of light at the nanoscale since they have zero group velocity while being much less damped than LSPRs. Finally, we envisage that the strong suppression of radiative losses herein discussed holds great promise for the development of high- $Q$ distributed feedback surfacepolariton lasers and plasmonic sensors with enhanced sensitivity.

We thank V. Giannini and M. C. Schaafsma for fruitful discussions, and M. Verschuuren and Y. Zhang for assistance in the fabrication of the samples. This work was supported by the Netherlands Foundation Fundamenteel Onderzoek der Materie (FOM) and the Nederlandse Organisatie voor Wetenschappelijk Onderzoek (NWO), and is part of an industrial partnership program between Philips and FOM.

[1] O. L. Muskens, V. Giannini, J. A. Sánchez-Gil, and J. Gómez Rivas, Strong Enhancement of the Radiative Decay Rate of Emitters by Single Plasmonic Nanoantennas, Nano Lett. 7, 2871 (2007).
[2] J. Aizpurua, G. W. Bryant, L. J. Richter, F. J. García de Abajo, B. K. Kelley, and T. Mallouk, Optical Properties of Coupled Metallic Nanorods for Field-Enhanced Spectroscopy, Phys. Rev. B 71, 235420 (2005).

[3] S. Palomba, M. Danckwerts, and Lukas Novotny, Nonlinear Plasmonics with Gold Nanoparticle Antennas, J. Opt. A 11, 114030 (2009).

[4] K. T. Carron, W. Fluhr, M. Meier, A. Wokaun, and H. W. Lehmann, Resonances of Two-dimensional Particle Gratings in Surface-Enhanced Raman Scattering, J. Opt. Soc. Am. B 3, 430 (1986).

[5] S. Zou, N. Janel, and G. C. Schatz, Silver Nanoparticle Array Structures that Produce Remarkably Narrow Plasmon Lineshapes, J. Chem. Phys. 120, 10871 (2004).

[6] S.I Zou and G.C. Schatz, Silver Nanoparticle Array Structures that Produce Giant Enhancements in Electromagnetic Fields, Chem. Phys. Lett. 403, 62 (2005).

[7] E. M. Hicks, S.Zou, G. C. Schatz, K. G. Spears, R. P. Van Duyne, L. Gunnarsson, T. Rindzevicius, B. Kasemo, and M. Käll, Controlling Plasmon Line Shapes through Diffractive Coupling in Linear Arrays of Cylyndircal Nanoparticles Fabricated by Electron Beam Lithography, Nano Lett. 5, 1065 (2005).

[8] B. Auguié and W. L. Barnes, Collective Resonances in Gold Nanoparticle Arrays, Phys. Rev. Lett. 101, 143902 (2008).

[9] Y. Chu, E. Schonbrun, T. Yang, and K. B. Crozier, Experimental Observation of Narrow Surface Plasmon Resonances in Gold Nanoparticle Arrays, Appl. Phys. Lett. 93, 181108 (2008).

[10] V. G. Kravets, F. Schedin, and A. N. Grigorenko, Extremely Narrow Plasmon Resonances Based on Diffraction Coupling of Localized Plasmons in Arrays of Metallic Nanoparticles, Phys. Rev. Lett. 101, 087403 (2008).

[11] G. Vecchi, V. Giannini, and J. Gómez Rivas, Shaping the Fluorescent Emission by Lattice Resonances in Plasmonic Crystals of Nanoantennas, Phys. Rev. Lett. 102, 146807 (2009).

[12] G. Vecchi, V. Giannini, and J. Gómez Rivas, Surface Modes in Plasmonic Crystals Induced by Diffractive Coupling of Nanoantennas, Phys. Rev. B 80, 201401 (2009).

[13] A. Bitzer, J. Wallauer, H. Merbold, H. Helm, T. Feurer, and M. Walther, Lattice Modes Mediate Radiative Coupling in Metamaterial Arrays, Opt. Express 17, 22108 (2009).

[14] V. Giannini, G. Vecchi, and J. Gómez Rivas, Lighting Up Multipolar Surface Plasmon Polaritons by Collective Resonances in Arrays of Nanoantennas, Phys. Rev. Lett. 105, 266801 (2010).

[15] P. Offermans, M. C. Schaafsma, S. R. K. Rodriguez, Y. Zhang, M. Crego-Calama, S.H. Brongersma, and J. Gómez Rivas, Universal Scaling of the Figure of Merit of Plasmonic Sensors, ACS Nano 5, 5151 (2011).

[16] S.C. Kitson, W.L. Barnes, and J.R. Sambles, Full Photonic Band Gap for Surface Modes in the Visible, Phys. Rev. Lett. 77, 2670 (1996).

[17] C. Sauvan, C. Billaudeau, S. Collin, N. Bardou, F. Pardo, J.-L. Pelouard, and Lalanne P., Surface Plasmon Coupling on Metallic Film Perforated by Two-Dimensional 
Rectangular Hole Array, Appl. Phys. Lett. 92, 011125 (2008).

[18] C. Ropers, D. J. Park, G. Stibenz, G. Steinmeyer, J. Kim, D.S. Kim, and C. Lienau, Femtosecond Light Transmission and Subradiant Damping in Plasmonic Crystals, Phys. Rev. Lett. 94, 113901 (2005).

[19] A. Ghoshal, I. Divliansky, and P. G. Kik, Experimental Observation of Mode-Selective Anticrossing in SurfacePlasmon-Coupled Metal Nanoparticle Arrays, Appl. Phys. Lett. 94, 171108 (2009).

[20] W. L. Barnes, T. W. Preist, S. C. Kitson, and J. R. Sambles, Physical Origin of Photonic Energy Gaps in the Propagation of Surface Plasmons on Gratings, Phys. Rev. B 54, 6227 (1996).

[21] M. A. Verschuuren, Substrate Conformal Imprint Lithography for Nanophotonics, Ph.D. thesis, Utrecht University, 2010.

[22] S. A. Maier, Plasmonics: Fundamentals and Applications (Springer, New York, USA, 2007).

[23] S. R. K. Rodriguez, O. T. A. Janssen, A. Abass, B. Maes, G. Vecchi, and J. Gómez Rivas, Opening Stop-Gaps in Plasmonic Crystals by Tuning the Radiative Coupling of Surface Plasmons to Diffracted Orders, arXiv:1110.3260.
[24] R.H. Dicke, Coherence in Sponateneous Radiation Processes, Phys. Rev. 93, 99 (1954).

[25] E. D. Palik, Handbook of Optical Constants of Solids (Academic Press, New York, 1985).

[26] U. Fano, Effects of Configuration Interaction on Intensities and Phase Shifts, Phys. Rev. 124, 1866 (1961).

[27] C. Genet, M. P. van Exter, and J. P. Woerdman, Fano-Type Interpretation of Red Shifts and Red Tails in Hole Array Transmission Spectra, Opt. Commun. 225, 331 (2003).

[28] M. Galli, S. L. Portalupi, M. Belotti, L. C. Andreani, L. O. Faolain, and T.F. Krauss, Light scattering and Fano Resonances in High-Q Photonic Crystal Nanocavities, Appl. Phys. Lett. 94, 071101 (2009).

[29] C.L. Garrido Alzar, M. A. G. Martinez, and P. Nussenzveig, Classical Analog of Electromagnetically Induced Transparency, Am. J. Phys. 70, 37 (2002).

[30] S. Mukherjee, H. Sobhani, J. Britt Lassiter, R. Bardhan, P. Nordlander, and N.J. Halas, Fanoshells: Nanoparticles with Built-in Fano Resonances, Nano Lett. 10, 2694 (2010).

[31] S. I. Bozhevolnyi and T. Søndergaard, General Properties of Slow-Plasmon Resonant Nanostructures: NanoAntennas and Resonators, Opt. Express 15, 10869 (2007). 